\title{
Minkowski Space-Time is Locally Extendible
}

\author{
John K. Beem* \\ Department of Mathematics, University of Missouri, Columbia, MO 65211, USA
}

\begin{abstract}
An example of a real analytic local extension of Minkowski spacetime is given in this note. This local extension is not across points of the $b$-boundary since Minkowski space-time has an empty $b$-boundary. Furthermore, this local extension is not across points of the causal boundary. The example indicates that the concept of local inextendibility may be less useful than originally envisioned.
\end{abstract}

\section{Extensions}

The subject of extensions is playing an important role in General Relativity. In particular, it is crucial to the study of singularities (cf. [1, 2]). In studying singularities, it is necessary to distinguish between true singularities which are irremovable and apparent singularities which arise merely because the given space-time is a proper subset of a larger space-time.

An extension of a space-time $(M, g)$ is a space-time $\left(M^{\prime}, g^{\prime}\right)$ and an isometry $f: M \rightarrow M^{\prime}$ which is onto a proper open subset of $M^{\prime}$. Since space-times are connected, the set $f(M)$ must have a nonempty boundary Bd $f(M)=$ closure $(f(M))-f(M)$. Simple arguments based on the fact that $\mathrm{Bd} f(M) \neq \emptyset$, show that an extendible space-time cannot be timelike, null or spacelike geodesically complete. A space-time with no extensions is said to be inextendible.

A local extension of the space-time $(M, g)$ is an open subset $U$ of $M$ which has noncompact closure $\bar{U}$ in $M$ and an extension $\left(U^{\prime}, g^{\prime}\right)$ of $(U, g \mid U)$ with isometry $f$ mapping $U$ into $U^{\prime}$ such that the image $f(U)$ has compact closure in $U^{\prime}$ (cf. [2, p. 59]). If $(M, g),\left(U^{\prime}, g^{\prime}\right)$ and $f: U \rightarrow U^{\prime}$ are all real analytic, then the local extension is said to be analytic.

Since closed subsets of a compact Hausdorff space are compact, a compact space-time is neither extendible nor locally extendible. Thus questions of extend-

* Partially supported by a grant from the Research Council of the Graduate School of the University of Missouri-Columbia 


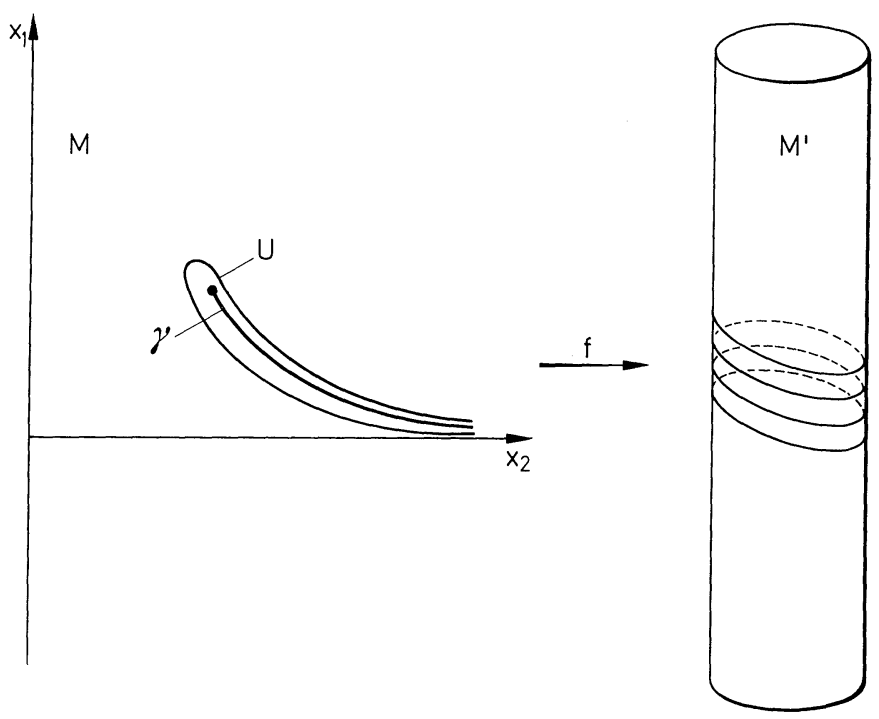

Fig. 1.

ibility are only meaningful in the noncompact case. A noncompact space-time $(M, g)$ which is extendible is trivially locally extendible because we may choose $U=M$ and $f=\mathrm{id}$. On the other hand, many space-times which are locally extendible fail to be extendible. In particular, Minkowski space-time is geodesically complete and hence inextendible but we will show that Minkowski spacetime admits local extensions.

Extensions and local extensions are usually thought of as enlargements which go across ideal boundary points of the given space-time. Accordingly, it will be useful to introduce the following notations. First, we will let $\partial_{c} M$ denote the causal boundary of $M$ formed using indecomposable past and future sets (cf. $[2,3]$ ). Second, let $\partial_{b} M$ denote the $b$-boundary of $M$ formed using a metric completion on the bundle of linear frames over $M$ (cf. $[2,4])$. If $(M, g)$ is Minkowski space-time, then $\partial_{c} M$ consists of $\mathscr{I}^{+}, \mathscr{I}^{-}, i^{+}, i^{-}$(cf. [2, p. 225]) and $\partial_{b} M=\emptyset$.

\section{A Local Extension of Minkowski Space-Time}

In this example, Minkowski space-time is shown to have an analytic local extension corresponding to a connected open subset with noncompact closure even though this space-time is geodesically complete and has no global extensions.

Example. Let $M=\mathbb{R}^{n}$ be given the usual Minkowskian metric $d s^{2}=-d x_{1}^{2}+d x_{2}^{2}$ $+\ldots+d x_{n}^{2}$. Let $M^{\prime}=\mathbb{R}^{1} \times T^{n-1}$ where $T^{n-1}=\left\{\left(\theta_{2}, \ldots, \theta_{n}\right) ; 0 \leqq \theta_{i} \leqq 1\right.$ for all $i=2, \ldots, n\}$ is the $(n-1)$-dimensional torus (using the usual identifications). The metric $g^{\prime}$ will be given by $\left(d s^{\prime}\right)^{2}=-d t^{2}+d \theta_{2}^{2}+d \theta_{3}^{2}+\ldots+d \theta_{n}^{2}$. Then $(M, g)$ is the universal covering space of $\left(M^{\prime}, g^{\prime}\right)$ and $f: M \rightarrow M^{\prime}$ given by $f\left(x_{1}, \ldots, x_{n}\right)$ $=\left(x_{1}, x_{2}(\bmod 1), \ldots, x_{n}(\bmod 1)\right)$ is a locally isometric covering map. Consider the curve $\gamma(s)=\left(s^{-1}, s, 0, \ldots, 0\right)$ in $M$ for $s \geqq 1$. The image of $\gamma$ under $f$ is a "spiral" which winds down towards the circle $t=\theta_{3}=\ldots=\theta_{n}=0$ in $M^{\prime}$. We wish to choose 
$U$ to be a "tube" about $\gamma$. In particular, we require $U \subset\left\{\left(x_{1}, \ldots, x_{n}\right) ; 0<x_{1}<2\right\}$ to be an open neighborhood of $\gamma$ such that $U$ is homeomorphic to $\mathbb{R}^{n}$ and such that $f \mid U$ is a homeomorphism of $U$ into $M^{\prime}$. It is thus necessary to have $U$ become thinner as $s \rightarrow \infty$, compare Fig. 1. While the set $U$ does not have compact closure, $f(U)$ does have compact closure because it lies in the compact subset $[0,2] \times T^{n-1}$ of $M^{\prime}$. Thus, Minkowski space-time has analytic local extensions corresponding to connected open subsets. Note also that in this example, the subset $U$ of $M$ about $\gamma$ approaches the ideal point $i^{0}$ which does not lie in $\partial_{c} M$. Thus the extension is neither across a point of $\partial_{c} M$ nor a point of $\partial_{b} M=\emptyset$.

This example shows that even geodesically complete space-times may admit local extensions. Furthermore, one must consider the possibility of local extensions which involve neither $\partial_{b} M$ nor $\partial_{c} M$.

As a last remark we note that some authors have considered a more restrictive notion of local extendibility by using $b$-incomplete curves with an endpoint in $\partial_{b} M$ (cf. $[1,5,6$, Chap. 5]). Since the $b$-boundary of Minkowski space-time is empty, there are no local extensions of Minkowski space-time of this more restricted type.

Acknowledgement. The author wishes to thank Paul E. Ehrlich for some very helpful discussions.

\section{References}

1. Ellis, G.F.R., Schmidt, B.G. : Gen. Rel. Grav. 8, 915 (1977)

2. Hawking, S.W., Ellis, G.F.R.: The large scale structure of space-time. Cambridge: Cambridge University Press 1973

3. Geroch, R., Kronheimer, E.H., Penrose, R.: Proc. R. Soc. Lond. Sect. A 327, 545 (1972)

4. Schmidt, B.G.: Gen. Rel. Grav. 1, 269 (1971)

5. Clarke, C.J.S.: Commun. Math. Phys. 32, 205 (1973)

6. Beem, J.K., Ehrlich, P.E.: The Lorentzian distance function and global Lorentzian geometry. New York, Basel: Marcel Dekker, to appear

Communicated by R. Geroch

Received September 14, 1979 
